L. Hesselholt

Nagoya Math. J.

Vol. 185 (2007), 93-109

\title{
ON THE $K$-THEORY OF THE COORDINATE AXES IN THE PLANE
}

\author{
LARS HESSELHOLT
}

\begin{abstract}
Let $k$ a regular noetherian $\mathbb{F}_{p}$-algebra, let $A=k[x, y] /(x y)$ be the coordinate ring of the coordinate axes in the affine $k$-plane, and let $I=(x, y)$ be the ideal that defines the intersection point. We evaluate the relative $K$ groups $K_{q}(A, I)$ completely in terms of the big de Rham-Witt groups of $k$. This generalizes a formula for $K_{1}(A, I)$ and $K_{2}(A, I)$ by Dennis and Krusemeyer.
\end{abstract}

\section{Introduction}

The algebraic $K$-groups of an algebraic variety encode deep arithmetic information about the variety. To wit, for non-singular varieties, the algebraic $K$-groups are related to the motivic cohomology groups by means of a spectral sequence

$$
E_{s, t}^{2}=H^{t-s}(X, \mathbb{Z}(t)) \Rightarrow K_{s+t}(X) .
$$

Indeed, this relationship was the basis of the original definition of motivic cohomology [1]. For singular varieties and non-reduced schemes, there is presently no definition of a motivic cohomology theory that relates to algebraic $K$-theory in this manner, but see Bloch-Esnault [2] and Rülling [19] for some work in this direction. However, it has recently become possible to evaluate the algebraic $K$-groups of some singular varieties and non-reduced schemes by using the cyclotomic trace map or Chern class map to topological cyclic homology [6]. We here use these methods to completely evaluate the algebraic $K$-groups of the coordinate axes in the plane. The groups in degrees less than or equal to two were evaluated twenty-five years ago by Dennis and Krusemeyer [5], but until now the groups in higher degrees have resisted calculation. We hope that this calculation will help in developing a motivic cohomology theory for singular varieties and non-reduced schemes.

Received August 9, 2005.

Revised November 15, 2005.

2000 Mathematics Subject Classification: Primary 19G50, 19G55; Secondary 11G20.

The author was partially supported by COE (Japan) and the National Science Foundation 
We now describe the results of this paper in more detail. Let $k$ be a ring, and let $A=k[x, y] /(x y)$ be the coordinate ring of the coordinate axes in the affine $k$-plane. The $K$-groups of $A$ decompose as the direct sum

$$
K_{q}(A)=K_{q}(k) \oplus K_{q}(A, I)
$$

of the $K$-groups of the ground ring $k$ and the relative $K$-groups of $A$ with respect to the ideal $I=(x, y)$. We evaluate the groups $K_{q}(A, I)$ completely in the case where $k$ is a regular $\mathbb{F}_{p}$-algebra. The result is stated in terms of the groups of big de Rham-Witt forms of $k$ as follows.

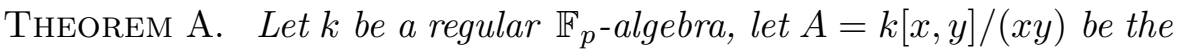
coordinate ring of the coordinate axes in the affine $k$-plane, and let $I \subset A$ be the ideal generated by $x$ and $y$. Then for all integers $q$, there is a canonical isomorphism

$$
K_{q}(A, I) \stackrel{\sim}{\leftarrow} \bigoplus_{m \geqslant 1} \mathbf{W}_{m} \Omega_{k}^{q-2 m}
$$

where $\mathbf{W}_{m} \Omega_{k}^{j}$ is the group of big de Rham-Witt $j$-forms of $k$.

The group $K_{2}(A, I)$ was evaluated by Dennis and Krusemeyer [5] twentyfive years ago. But it was previously known only that the higher relative $K$-groups are $p$-primary torsion groups [20]. The group of big de RhamWitt $j$-forms $\mathbf{W}_{n} \Omega_{k}^{j}$ was introduced in [14, Def. 1.1.6]. It decomposes as a product of the more familiar $p$-typical de Rham-Witt $j$-forms $W_{s} \Omega_{k}^{j}$ defined by Bloch-Deligne-Illusie [16]. Indeed, by [14, Cor. 1.2.6] there is a canonical isomorphism

$$
\mathbf{W}_{m} \Omega_{k}^{j} \stackrel{\sim}{\longrightarrow} \prod_{d} W_{s} \Omega_{k}^{j}
$$

where the product ranges over all integers $1 \leqslant d \leqslant m$ that are not divisible by $p$, and where $s=s(m, d)$ is the unique positive integer with $p^{s-1} d \leqslant m<$ $p^{s} d$. The structure of the groups $W_{s} \Omega_{k}^{j}$ is well-understood by [16, I.3.9]. For example, $W_{s} \Omega_{\mathbb{F}_{p}}^{j}$ is canonically isomorphic to $\mathbb{Z} / p^{s} \mathbb{Z}$, for $j=0$, and is equal to zero, for $j>0$.

Let $B=k[x] \times k[y]$ be the normalization of the ring $A$, and let $K(A, B, I)$ be the bi-relative $K$-theory spectrum defined to be the iterated mapping fiber of the following diagram of $K$-theory spectra.

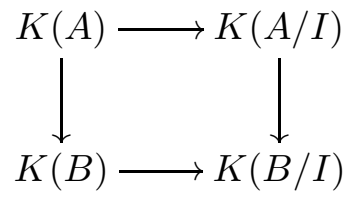


The lower horizontal map in this diagram is a weak equivalence since the ring $k$ is regular. It follows that also the canonical map

$$
K(A, B, I) \rightarrow K(A, I)
$$

is a weak equivalence. We proved recently in $[6$, Thm. A] that for every prime $p$, the cyclotomic trace map induces an isomorphism

$$
K_{q}\left(A, B, I, \mathbb{Z} / p^{v}\right) \stackrel{\sim}{\longrightarrow} \mathrm{TC}_{q}\left(A, B, I ; p, \mathbb{Z} / p^{v}\right)
$$

and it is the bi-relative topological cyclic homology groups on the righthand side that we evaluate here. The method is similar to the calculation of the topological cyclic homology of the ring of dual numbers by the author and Madsen [13, 14]. We first prove a general formula that expresses the bi-relative topological cyclic homology groups above in terms of the $R O(\mathbb{T})$ graded equivariant homotopy groups

$$
\operatorname{TR}_{\alpha}^{n}(k ; p)=\left[S^{\alpha} \wedge\left(\mathbb{T} / C_{p^{n-1}}\right)_{+}, T(k)\right]_{\mathbb{T}}
$$

of the topological Hochschild $\mathbb{T}$-spectrum $T(k)$. Here $\mathbb{T}$ is the circle group and $C_{r} \subset \mathbb{T}$ is the subgroup of order $r$. To state the formula, which is valid for any ring $k$, we let $\lambda_{i}$ be the complex $\mathbb{T}$-representation $\mathbb{C}(1) \oplus \cdots \oplus \mathbb{C}(i)$.

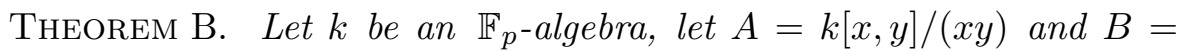
$k[x] \times k[y]$, and let $I$ be the common ideal of $A$ and $B$ generated by $x$ and $y$. Then for all integers $q$, there is a canonical isomorphism

$$
\operatorname{TC}_{q}(A, B, I ; p) \stackrel{\sim}{\longrightarrow} \prod \lim _{R} \operatorname{TR}_{q-\lambda_{p^{r-1} d}}^{r}(k ; p)
$$

where the product ranges over the positive integers $d$ that are not divisible by $p$. The analogous statement for the groups with $\mathbb{Z} / p^{v}$-coefficients is valid for any ring $k$.

The limit system on the right-hand side of the statement of Thm. B stabilizes in the sense that the structure map

$$
R: \mathrm{TR}_{q-\lambda_{p^{r-1} d}}^{r}(k ; p) \rightarrow \mathrm{TR}_{q-\lambda_{p^{r-2} d}}^{r-1}(k ; p)
$$

is an isomorphism for $q<\operatorname{dim}_{\mathbb{R}}\left(\lambda_{p^{r-1} d}\right)$. See Lemma 2.3 below.

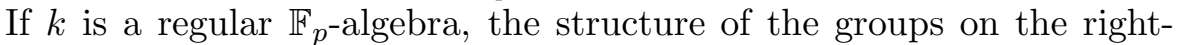
hand side of the statement of Thm. B was determined in [14, Thm. 2.2.2]; 
see also [11, Thm. 16]. We recall the result in Sect. 3 below and complete the proof of Thm. A.

Finally, we mention that the analog of Thm. A for $k$ a regular $\mathbb{Q}$-algebra is known. Indeed, by a recent theorem of Cortiñas [4, Thm. 0.1] (which inspired us to prove $[6, \mathrm{Thm} . \mathrm{A}]$ ), the trace map induces an isomorphism

$$
K_{q}(A, B, I) \otimes \mathbb{Q} \stackrel{\sim}{\longrightarrow} \mathrm{HC}_{q}^{-}(A \otimes \mathbb{Q}, B \otimes \mathbb{Q}, I \otimes \mathbb{Q})
$$

and the bi-relative negative cyclic homology groups on the right-hand side were evaluated long ago by Geller, Reid, and Weibel [7, 8]. The result is that, if $k$ is a regular $\mathbb{Q}$-algebra, then there is a canonical isomorphism

$$
K_{q}(A, I) \stackrel{\sim}{\longleftarrow} \bigoplus_{m \geqslant 1} \Omega_{k}^{q-2 m}
$$

where $\Omega_{k}^{j}$ is the group of absolute Kähler $j$-forms of $k$. This formula differs from the formula of Thm. A in degrees $q \geqslant 4$. Indeed, the group $K_{4}(A, I)$ is isomorphic to $\Omega_{k}^{2} \oplus k$, if $k$ is a regular $\mathbb{Q}$-algebra, to $\Omega_{k}^{2} \oplus W_{2}(k)$, if $k$ is a regular $\mathbb{F}_{2}$-algebra, and to $\Omega_{k}^{2} \oplus k \oplus k$, if $k$ is a regular $\mathbb{F}_{p}$-algebra and $p>2$.

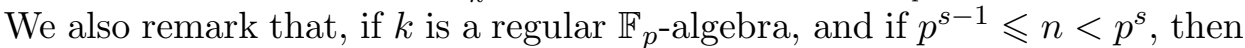
the group $K_{2 n}(A, I)$ has exponent exactly $p^{s}$.

The result of Thm. A was announced in [6, Thm. C].

All rings considered in this paper are assumed to be commutative. We write $\mathbb{N}$ and $I_{p}$ for the sets of positive integers and positive integers prime to $p$, respectively. We say that a map of $\mathbb{T}$-spectra is an $\mathcal{F}$-equivalence if the induced map of $C$-fixed point spectra is a weak equivalence, for all finite subgroups $C \subset \mathbb{T}$.

Finally, the author would like to thank an anonymous referee for a very careful reading of an earlier version of this paper and for a number of helpful suggestions on improving the exposition.

\section{$\S 1$. Topological Hochschild homology}

The proof of Thm. B of the introduction is based on a description of the bi-relative topological Hochschild $\mathbb{T}$-spectrum $T(A, B, I)$ defined to be the iterated mapping fiber of the following diagram of topological Hochschild $\mathbb{T}$-spectra.

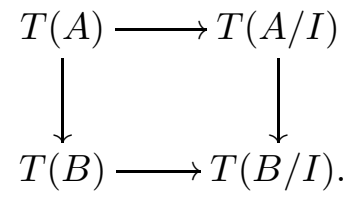


We refer to [11] for an introduction to topological Hochschild and cyclic homology and for further references. In this section, we will prove the following result.

Proposition 1.2. Let $k$ be any ring, let $A=k[x, y] /(x y)$ and $B=$ $k[x] \times k[y]$, and let $I$ be the common ideal generated by $x$ and $y$. Then there is a canonical $\mathcal{F}$-equivalence of $\mathbb{T}$-spectra

$$
\bigvee_{i \in \mathbb{N}} T(k) \wedge S^{\lambda_{i}} \wedge\left(\mathbb{T} / C_{i}\right)_{+}[1] \stackrel{\sim}{\longrightarrow} T(A, B, I)
$$

where, on the left-hand side, [1] indicates desuspension.

The rings $B=k[x] \times k[y]$ and $B / I=k \times k$ are both product rings. Moreover, topological Hochschild homology preserves products of rings in the sense that for every pair of rings $R$ and $S$, the canonical map of $\mathbb{T}$-spectra

$$
T(R \times S) \rightarrow T(R) \times T(S)
$$

is an $\mathcal{F}$-equivalence [3, Prop. 4.20]. Hence, the canonical map from $T(A, B, I)$ to the iterated mapping fiber of the following diagram of $\mathbb{T}$-spectra is an $\mathcal{F}$-equivalence.

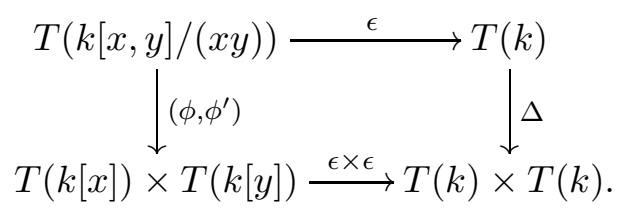

The rings that occur in this diagram are all pointed monoid algebras. By definition, a pointed monoid $\Pi$ is a monoid in the symmetric monoidal category of pointed sets and smash product, and the pointed monoid algebra $k(\Pi)$ is the quotient of the monoid algebra $k[\Pi]$ by the ideal generated by the base-point of $\Pi$. The diagram of rings (1.3) is then induced from the diagram of pointed monoids

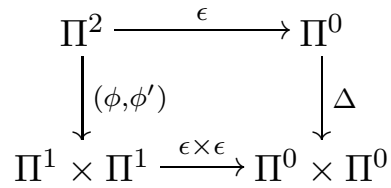

where $\Pi^{0}=\{0,1\}$ with base-point 0 , where $\Pi^{1}=\left\{0,1, z, z^{2}, \ldots\right\}$ with base-point 0 , and where $\Pi^{2}=\left\{0,1, x, x^{2}, \ldots, y, y^{2}, \ldots\right\}$ with base-point 0 
and with multiplication given by $x y=0$. The map $\phi$ (resp. $\phi^{\prime}$ ) takes the variables $x$ and $y$ to $z$ and 0 (resp. to 0 and $z$ ), and the maps labeled $\epsilon$ take the variables $x, y$, and $z$ to 1 .

The topological Hochschild $\mathbb{T}$-spectrum of a pointed monoid algebra $k(\Pi)$ decomposes, up to $\mathcal{F}$-equivalence, as the smash product

$$
T(k) \wedge N^{\mathrm{cy}}(\Pi) \stackrel{\sim}{\longrightarrow} T(k(\Pi))
$$

of the topological Hochschild $\mathbb{T}$-spectrum of the coefficient ring $k$ and the cyclic bar-construction of the pointed monoid $\Pi$. This is proved in $[13$, Thm. 7.1] but see also [11, Prop. 4]. The cyclic bar-construction is the geometric realization of the pointed cyclic set with $m$-simplices

$$
N^{\mathrm{cy}}(\Pi)[m]=\Pi \wedge \cdots \wedge \Pi \quad(m+1 \text { factors })
$$

and with the Hochschild-type cyclic structure maps

$$
\begin{array}{rlrl}
d_{i}\left(\pi_{0} \wedge \cdots \wedge \pi_{m}\right) & =\pi_{0} \wedge \cdots \wedge \pi_{i} \pi_{i+1} \wedge \cdots \wedge \pi_{m}, & & 0 \leqslant i<m, \\
& =\pi_{m} \pi_{0} \wedge \pi_{1} \wedge \cdots \wedge \pi_{m-1}, & & i=m, \\
s_{i}\left(\pi_{0} \wedge \cdots \wedge \pi_{m}\right) & =\pi_{0} \wedge \cdots \wedge \pi_{i} \wedge 1 \wedge \pi_{i+1} \wedge \cdots \wedge \pi_{m}, & & 0 \leqslant i \leqslant m, \\
t_{m}\left(\pi_{0} \wedge \cdots \wedge \pi_{m}\right) & =\pi_{m} \wedge \pi_{0} \wedge \pi_{1} \wedge \cdots \wedge \pi_{m-1} . &
\end{array}
$$

It is a pointed $\mathbb{T}$-space by the theory of cyclic sets [18, 7.1.9]. It follows that the $\mathbb{T}$-spectrum $T(A, B, I)$ is canonically $\mathcal{F}$-equivalent to the iterated mapping fiber of the following diagram of $\mathbb{T}$-spectra.

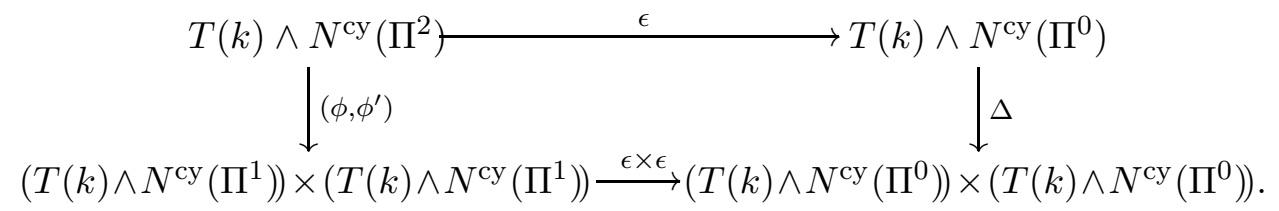

The cyclic bar-constructions of $\Pi^{1}$ and $\Pi^{2}$ have natural wedgedecompositions which we now explain.

We define $N^{\text {cy }}\left(\Pi^{1}, i\right)[m]$ to be the subset of $N^{\text {cy }}\left(\Pi^{1}\right)[m]$ that consists of the base-point and of the simplices $z^{i_{0}} \wedge \cdots \wedge z^{i_{m}}$ with $i_{0}+\cdots+i_{m}=i$. It is clear that the pointed set $N^{\mathrm{cy}}\left(\Pi^{1}\right)[m]$ decomposes as the wedge-sum of the pointed subsets $N^{\mathrm{cy}}\left(\Pi^{1}, i\right)[m]$ where $i$ ranges over the non-negative integers. The cyclic structure maps preserve this decomposition, and hence, 
the geometric realization decomposes accordingly as a wedge-sum of pointed $\mathbb{T}$-spaces indexed by the non-negative integers.

$$
N^{\mathrm{cy}}\left(\Pi^{1}\right)=\bigvee N^{\mathrm{cy}}\left(\Pi^{1}, i\right)
$$

To state the analogous wedge-decomposition of $N^{\text {cy }}\left(\Pi^{2}\right)$, we first recall the notion of a cyclical word.

A word of length $m$ with letters in a set $S$ is a function

$$
\omega:\{1,2, \ldots, m\} \rightarrow S .
$$

The action by the cyclic group $C_{m}$ of order $m$ on the set $\{1,2, \ldots, m\}$ by cyclic permutation induces an action on the set of words of length $m$ in $S$. A cyclical word of length $m$ with letters in $S$ is an orbit for the action of $C_{m}$ on the set of words of length $m$ in $S$. We write $\bar{\omega}$ for the orbit through $\omega$. By the period of $\bar{\omega}$, we mean the length of the orbit $\bar{\omega}$. In particular, the set that consists of the empty word is a cyclical word of length 0 and period 1 .

We associate a word $\omega(\pi)$ with letters in $x$ and $y$ to every non-zero element $\pi \in \Pi^{2}$. A non-zero element $\pi \in \Pi^{2}$ is either of the form $\pi=x^{i}$ or $\pi=y^{i}$. In the former case, we define $\omega(\pi)$ to be the unique word of length $i$ all of whose letters are $x$, and in the latter case, we define $\omega(\pi)$ to be the unique word of length $i$ all of whose letters are $y$. More generally, we associate to every $(m+1)$-tuple $\left(\pi_{0}, \ldots, \pi_{m}\right)$ of non-zero elements of $\Pi$ the word

$$
\omega\left(\pi_{0}, \ldots, \pi_{m}\right)=\omega\left(\pi_{0}\right) * \cdots * \omega\left(\pi_{m}\right)
$$

defined to be the concatenation of the words $\omega\left(\pi_{0}\right), \ldots, \omega\left(\pi_{m}\right)$. Now, for every cyclical word $\bar{\omega}$ with letters $x$ and $y$, we define

$$
N^{\mathrm{cy}}\left(\Pi^{2}, \bar{\omega}\right)[m] \subset N^{\mathrm{cy}}\left(\Pi^{2}\right)[m]
$$

to be the subset that consists of the base-point and the elements $\pi_{0} \wedge \cdots \wedge \pi_{m}$, where $\left(\pi_{0}, \ldots, \pi_{m}\right)$ ranges over all $(m+1)$-tuples of non-zero elements of $\Pi^{2}$ such that $\omega\left(\pi_{0}, \ldots, \pi_{m}\right) \in \bar{\omega}$. As $m \geqslant 0$ varies, these subsets form a cyclic subset

$$
N^{\mathrm{cy}}\left(\Pi^{2}, \bar{\omega}\right)[-] \subset N^{\mathrm{cy}}\left(\Pi^{2}\right)[-],
$$

and we define $N^{\text {cy }}\left(\Pi^{2}, \bar{\omega}\right) \subset N^{\text {cy }}\left(\Pi^{2}\right)$ to be the geometric realization. It is clear that the cyclic set $N^{\mathrm{cy}}\left(\Pi^{2}\right)[-]$ decomposes as the wedge-sum of the 
cyclic subsets $N^{\text {cy }}\left(\Pi^{2}, \bar{\omega}\right)[-]$, where $\bar{\omega}$ ranges over all cyclical words with letters $x$ and $y$. Hence, the geometric realization decomposes as a wedgesum of pointed $\mathbb{T}$-spaces

$$
N^{\mathrm{cy}}\left(\Pi^{2}\right)=\bigvee N^{\mathrm{cy}}\left(\Pi^{2}, \bar{\omega}\right)
$$

indexed by all cyclical words with letters $x$ and $y$.

Lemma 1.5. There is a canonical $\mathcal{F}$-equivalence of $\mathbb{T}$-spectra

$$
\bigvee T(k) \wedge N^{\mathrm{cy}}\left(\Pi^{2}, \bar{\omega}\right) \stackrel{\sim}{\longrightarrow} T(A, B, I),
$$

where the wedge-sum on the left-hand side ranges over all cyclical words of period $s \geqslant 2$ with letters $x$ and $y$.

Proof. Let $\bar{\omega}$ be a cyclical word of period $s \geq 2$. Then every word $\omega \in \bar{\omega}$ has both of the letters $x$ and $y$. Therefore, the compositions of the canonical map

$$
T(k) \wedge N^{\mathrm{cy}}\left(\Pi^{2}, \bar{\omega}\right) \rightarrow T(A)
$$

and the maps $\phi: T(A) \rightarrow T(B), \phi^{\prime}: T(A) \rightarrow T(B)$, and $\epsilon: T(A) \rightarrow T(A / I)$ are all equal to the constant map. Hence, we obtain a canonical map of $\mathbb{T}$-spectra

$$
T(k) \wedge N^{\mathrm{cy}}\left(\Pi^{2}, \bar{\omega}\right) \rightarrow T(A, B, I)
$$

and the wedge-sum of these maps constitute the map of the statement. The diagram (1.3) and the $\mathcal{F}$-equivalence (1.4) show that this map is an $\mathcal{F}$-equivalence.

Lemma 1.6. Let $\bar{\omega}$ be a cyclical word of period $s \geqslant 2$ with letters $x$ and $y$. The homotopy type of the pointed $\mathbb{T}$-space $N^{\mathrm{cy}}\left(\Pi^{2}, \bar{\omega}\right)$ is given as follows.

(i) If $\bar{\omega}$ has period $s=2$ and length $m=2 i$, then a choice of representative word $\omega \in \bar{\omega}$ determines a $\mathbb{T}$-equivariant homeomorphism

$$
S^{\mathbb{R}\left[C_{m}\right]-1} \wedge_{C_{i}} \mathbb{T}_{+} \stackrel{\sim}{\longrightarrow} N^{\mathrm{cy}}\left(\Pi^{2}, \bar{\omega}\right),
$$

where $\mathbb{R}\left[C_{m}\right]-1$ is the reduced regular representation of $C_{m}$.

(ii) If $\bar{\omega}$ has period $s>2$, then $N^{\mathrm{cy}}\left(\Pi^{2}, \bar{\omega}\right)$ is $\mathbb{T}$-equivariantly contractible. 
Proof. We refer the reader to [18] for the basic theory of cyclic sets and their geometric realization. Let $\bar{\omega}$ be a cyclical word of period $s \geq 2$ and length $m=s i$ with letters $x$ and $y$, and let $\omega \in \bar{\omega}$. We let $\left(\pi_{0}, \ldots, \pi_{m-1}\right)$ be the unique $m$-tuple of non-zero elements in $\Pi^{2}$ such that $\omega\left(\pi_{0}, \ldots, \pi_{m-1}\right)=$ $\omega$. Then the pointed cyclic set $N^{\mathrm{cy}}\left(\Pi^{2}, \bar{\omega}\right)[-]$ is generated by the $(m-1)$ simplex $\pi_{0} \wedge \cdots \wedge \pi_{m-1}$. Hence, there is a unique surjective map of pointed cyclic sets

$$
f_{\omega}: \Lambda^{m-1}[-]_{+} \rightarrow N^{\mathrm{cy}}\left(\Pi^{2}, \bar{\omega}\right)[-]
$$

that maps the canonical generator of the cyclic standard $(m-1)$-simplex to the generator $\pi_{0} \wedge \cdots \wedge \pi_{m-1}$. We recall that the automorphism group of the pointed cyclic set $\Lambda^{m-1}[-]_{+}$is a cyclic group of order $m$ generated by the dual of the cyclic operator $t_{m}$. Since the cyclic operator $t_{m}^{s}$ fixes the generator $\pi_{0} \wedge \cdots \wedge \pi_{m-1}$, we obtain a factorization of the map $f_{\omega}$ over the quotient by the subgroup of the automorphism group or order $i=\mathrm{m} / \mathrm{s}$,

$$
f_{\omega}:\left(\Lambda^{m-1}[-] / C_{i}\right)_{+} \rightarrow N^{\mathrm{cy}}\left(\Pi^{2}, \bar{\omega}\right)[-] .
$$

We next recall that the geometric realization of the cyclic standard $(m-1)$ simplex is $\mathbb{T}$-equivariantly homeomorphic to $\Delta^{m-1} \times \mathbb{T}$, where $\mathbb{T}$ acts by multiplication in the second factor. Moreover, the homeomorphism may be chosen in such that the dual of the cyclic operator $t_{m}$ acts on $\Delta^{m-1}$ by the affine map that cyclically permutes the vertices and on $\mathbb{T}$ by rotation through $2 \pi / m$; see [13, Sect. 7.2$]$. It follows that the map $f_{\omega}$ gives rise to a continuous $\mathbb{T}$-equivariant surjection

$$
f_{\omega}:\left(\Delta^{m-1} \times_{C_{i}} \mathbb{T}\right)_{+} \rightarrow N^{\mathrm{cy}}\left(\Pi^{2}, \bar{\omega}\right) .
$$

There is a canonical $C_{i}$-equivariant homeomorphism

$$
\Delta^{s-1} * \cdots * \Delta^{s-1} \stackrel{\sim}{\longrightarrow} \Delta^{m-1},
$$

where the group $C_{i}$ cyclically permutes the $i$ factors in the join on the left-hand side, and where the map $f_{\omega}$ collapses the join of a number of codimension 1 faces of $\Delta^{s-1}$ to the base-point. If the period of $\bar{\omega}$ is $s=2$, then the map $f_{\omega}$ exactly collapses the whole boundary $\partial \Delta^{m-1} \subset \Delta^{m-1}$ to the base-point. Hence, in this case, we have a $\mathbb{T}$-equivariant homeomorphism

$$
f_{\omega}:\left(\Delta^{m-1} / \partial \Delta^{m-1}\right) \wedge_{C_{i}} \mathbb{T}_{+} \stackrel{\sim}{\longrightarrow} N^{\mathrm{cy}}\left(\Pi^{2}, \bar{\omega}\right) .
$$

The simplex $\Delta^{m-1}$ embeds as the convex hull of the group elements in the regular representation $\mathbb{R}\left[C_{m}\right]$. This identifies the $C_{m^{-}}$space $\Delta^{m-1} / \partial \Delta^{m-1}$ 
with the one-point compactification of the reduced regular representation $\mathbb{R}\left[C_{m}\right]-1$ as stated. This completes the proof of the statement for $s=2$. If the period $s>2$, then there exists a codimension 1 face $F \subset \Delta^{s-1}$ that is not collapsed to the base-point. We have a canonical homeomorphism

$$
\operatorname{cone}(F) \stackrel{\sim}{\longrightarrow} \Delta^{s-1}
$$

of the unreduced cone on the face $F$ onto the simplex $\Delta^{s-1}$. The canonical null-homotopy of the unreduced cone induces a $C_{i}$-equivariant nullhomotopy

$$
\Delta^{s-1} * \cdots * \Delta^{s-1} \times[0,1] \rightarrow \Delta^{s-1} * \cdots * \Delta^{s-1},
$$

and since the face $F$ is not collapsed to the base-point by the map $f_{\omega}$, this induces a $\mathbb{T}$-equivariant null-homotopy

$$
N^{\mathrm{cy}}\left(\Pi^{2}, \bar{\omega}\right) \wedge[0,1]_{+} \rightarrow N^{\mathrm{cy}}\left(\Pi^{2}, \bar{\omega}\right) .
$$

This completes the proof of the statement for $s>2$.

Remark 1.7. The statement of Lemma 1.6 may be viewed as a topological refinement of the calculation in [9] of the Hochschild homology of the pointed monoid ring $\mathbb{Z}\left(\Pi^{2}\right)=\mathbb{Z}[x, y] /(x y)$. Indeed, for any pointed monoid $\Pi$, the reduced singular homology groups $\tilde{H}_{*}\left(N^{\text {cy }}(\Pi) ; \mathbb{Z}\right)$ and the Hochschild homology groups $\mathrm{HH}_{*}(\mathbb{Z}(\Pi))$ are canonically isomorphic.

Proof of Proposition 1.2. It follows from Lemmas 1.5 and 1.6 that we have an $\mathcal{F}$-equivalence of $\mathbb{T}$-spectra

$$
\bigvee T(k) \wedge S^{\mathbb{R}\left[C_{2 i}\right]-1} \wedge_{C_{i}} \mathbb{T}_{+} \stackrel{\sim}{\longrightarrow} T(A, B, I)
$$

where the wedge sum ranges over all positive integers $i$. The equivalence depends on a choice, for every cyclical word $\bar{\omega}$ with letters $x$ and $y$ of period 2, of a representative word $\omega \in \bar{\omega}$. We choose the representative $\omega=x y \ldots x y$. Now, as a representation of the subgroup $C_{i} \subset C_{2 i}$, the regular representation $\mathbb{R}\left[C_{2 i}\right]$ is isomorphic to the complex representation $\lambda_{i}=\mathbb{C}(1) \oplus \cdots \oplus \mathbb{C}(i)$, where $\mathbb{C}(t)$ denotes the representation of $\mathbb{T}$ on $\mathbb{C}$ through the $t$-fold power map. Hence, a choice of such an isomorphism determines a $\mathbb{T}$-equivariant homeomorphism

$$
S^{\lambda_{i}} \wedge_{C_{i}} \mathbb{T}_{+} \stackrel{\sim}{\longrightarrow} S^{\mathbb{R}\left[C_{2 i}\right]} \wedge_{C_{i}} \mathbb{T}_{+}
$$


Moreover, since the $C_{i}$-action on $\lambda_{i}$ extends to a $\mathbb{T}$-action, we further have the canonical $\mathbb{T}$-equivariant homeomorphism

$$
S^{\lambda_{i}} \wedge\left(\mathbb{T} / C_{i}\right)_{+} \stackrel{\sim}{\longrightarrow} S^{\lambda_{i}} \wedge_{C_{i}} \mathbb{T}_{+}
$$

that takes $\left(w, z C_{i}\right)$ to the class of $\left(z^{-1} w, z\right)$. The completes the proof.

\section{$\S 2$. Topological cyclic homology}

In this section, we prove the formula for the bi-relative topological cyclic homology groups $\mathrm{TC}_{q}(A, B, I ; p)$ that was stated in $\mathrm{Thm}$. B of the introduction. We derive this formula from the corresponding formula for topological Hochschild homology that we proved in Prop. 1.2 above. The argument is very similar to the analogous argument in the case of truncated polynomial algebras $[12,14,11]$. We refer the reader to $[11,3.7]$ for the definition of topological cyclic homology.

We have from Prop. 1.2 an $\mathcal{F}$-equivalence of $\mathbb{T}$-spectra

$$
\bigvee_{i \in \mathbb{N}} T(k) \wedge S^{\lambda_{i}} \wedge\left(\mathbb{T} / C_{i}\right)_{+}[1] \stackrel{\sim}{\rightarrow} T(A, B, I),
$$

and we wish to evaluate the homotopy groups of the $C_{p^{n-1}}$-fixed point spectra. To this end, we reindex the wedge-sum on the left-hand side after the $p$-adic valuation of $i \in \mathbb{N}$. The left-hand side is then rewritten as

$$
\begin{aligned}
& \bigvee_{d \in \mathbb{N}} T(k) \wedge S^{\lambda_{p^{n-1}}} \wedge\left(\mathbb{T} / C_{p^{n-1} d}\right)_{+}[1] \\
& \vee \bigvee_{r=1}^{n-1} \bigvee_{d \in I_{p}} T(k) \wedge S^{\lambda_{p^{r-1}}} \wedge\left(\mathbb{T} / C_{p^{r-1} d}\right)_{+}[1]
\end{aligned}
$$

where $\mathbb{N}$ and $I_{p}$ are the sets of positive integers and positive integers that are not divisible by $p$, respectively. Hence, the $\mathbb{T}$-spectrum $\rho_{p^{n-1}}^{*} T(A, B, I)^{C_{p^{n-1}}}$ is equivalent to the wedge-sum

$$
\begin{aligned}
& \bigvee_{d \in \mathbb{N}} \rho_{p^{n-1}}^{*}\left(T(k) \wedge S^{\lambda_{p^{n-1} d}} \wedge\left(\mathbb{T} / C_{p^{n-1} d}\right)_{+}\right)^{C_{p^{n-1}}[1]} \\
& \vee \bigvee_{r=1}^{n-1} \bigvee_{d \in I_{p}} \rho_{p^{n-r}}^{*}\left(\rho_{p^{r-1}}^{*}\left(T(k) \wedge S^{\lambda_{p^{r-1}}} \wedge\left(\mathbb{T} / C_{p^{r-1} d}\right)_{+}\right)^{C_{p^{r-1}}}\right)^{C_{p^{n-r}}}[1]
\end{aligned}
$$

Now, for every $\mathbb{T}$-spectrum $T$, there is a natural equivalence of $\mathbb{T}$-spectra

$$
\rho_{p^{m}}^{*} T^{C_{p^{m}}} \wedge \rho_{p^{m}}^{*}\left(\left(\mathbb{T} / C_{p^{m}}\right)_{+}\right)^{C_{p^{m}}} \stackrel{\sim}{\longrightarrow} \rho_{p^{m}}^{*}\left(T \wedge\left(\mathbb{T} / C_{p^{m}}\right)_{+}\right)^{C_{p^{m}}}
$$


and the $p^{m}$ th root map defines a $\mathbb{T}$-equivariant homeomorphism

$$
\left(\mathbb{T} / C_{d}\right)_{+} \stackrel{\sim}{\rightarrow} \rho_{p^{m}}^{*}\left(\left(\mathbb{T} / C_{p^{m} d}\right)_{+}\right)^{C_{p^{m}}} .
$$

Hence, we can rewrite the wedge-sum above as follows.

$$
\begin{aligned}
& \bigvee_{d \in \mathbb{N}} \rho_{p^{n-1}}^{*}\left(T(k) \wedge S^{\lambda_{p^{n-1}}}\right)^{C_{p^{n-1}}} \wedge\left(\mathbb{T} / C_{d}\right)_{+}[1] \\
& \vee \bigvee_{r=1}^{n-1} \bigvee_{d \in I_{p}} \rho_{p^{n-r}}^{*}\left(\rho_{p^{r-1}}^{*}\left(T(k) \wedge S^{\lambda_{p^{r-1}}}\right)^{C_{p^{r-1}}} \wedge\left(\mathbb{T} / C_{d}\right)_{+}\right)^{C_{p^{n-r}}}[1]
\end{aligned}
$$

We recall from [15, Lemma 3.4.1] that if $T$ is a $\mathbb{T}$-spectrum, if $d \in I_{p}$, and if $\iota:\left\{C_{d}\right\} \rightarrow \mathbb{T} / C_{d}$ is the canonical inclusion, then the map

$$
V^{m} \iota_{*}+d V^{m} \iota_{*}: \pi_{q}(T) \oplus \pi_{q-1}(T) \rightarrow \pi_{q}\left(\rho_{p^{m}}^{*}\left(T \wedge\left(\mathbb{T} / C_{d}\right)_{+}\right)^{C_{p^{m}}}\right)
$$

is an isomorphism. It follows that the group $\operatorname{TR}_{q}^{n}(A, B, I ; p)$ is canonically isomorphic to the sum

$$
\begin{aligned}
& \bigoplus_{d \in \mathbb{N}}\left(\operatorname{TR}_{q+1-\lambda_{p^{n-1} d}}^{n}(k ; p) \oplus \operatorname{TR}_{q-\lambda_{p^{n-1} d}}^{n}(k ; p)\right) \\
& \oplus \bigoplus_{r=1}^{n-1} \bigoplus_{d \in I_{p}}\left(\operatorname{TR}_{q+1-\lambda_{p^{r-1} d}}^{r}(k ; p) \oplus \mathrm{TR}_{q-\lambda_{p^{r-1} d}}^{r}(k ; p)\right) .
\end{aligned}
$$

We consider the groups $\operatorname{TR}_{q}^{n}(A, B, I ; p)$ for varying $n \geqslant 1$ as a pro-abelian group whose structure map is the Frobenius map

$$
F: \operatorname{TR}_{q}^{n}(A, B, I ; p) \rightarrow \mathrm{TR}_{q}^{n-1}(A, B, I ; p) .
$$

The Frobenius map takes the summand with index $d \in \mathbb{N}$ in the top line of (2.1) for $n$ to the summand with index $p d \in \mathbb{N}$ in the top line of (2.1) for $n-1$. It takes the summand with indices $d \in I_{p}$ and $1 \leqslant r<n-1$ in the bottom line of (2.1) for $n$ to the summand with the same indices in the bottom line of (2.1) for $n-1$. Finally, it takes the summand with indices $d \in I_{p}$ and $r=n-1$ in the bottom line of (2.1) for $n$ to the summand with index $d \in \mathbb{N}$ in the top line of (2.1) for $n-1$. It follows that the sub-pro-abelian group of $\operatorname{TR}_{q}^{n}(A, B, I ; p)$ given by the top line of (2.1) is Mittag-Leffler zero, since the sum in (2.1) is finite. Hence, the projection onto the quotient pro-abelian group of $\operatorname{TR}_{q}^{n}(A, B, I ; p)$ given by the bottom 
line of (2.1) is an isomorphism of pro-abelian groups. The value of the Frobenius map on the bottom line of (2.1) follows immediately from the relations $F V=p$ and $F d V=d$. Indeed, the Frobenius preserves the direct sum decomposition and restricts to the maps

$$
\begin{gathered}
F=p: \operatorname{TR}_{q+1-\lambda}^{r}(k ; p) \rightarrow \mathrm{TR}_{q+1-\lambda}^{r}(k ; p), \\
F=\mathrm{id}: \operatorname{TR}_{q-\lambda}^{r}(k ; p) \rightarrow \mathrm{TR}_{q-\lambda}^{r}(k ; p),
\end{gathered}
$$

respectively, on the first and second summand of the bottom line of (2.1).

We now assume that the group $\operatorname{TR}_{q-\lambda}^{r}(k ; p)$ is annihilated by $p^{m}$, for some $m$. If $k$ is an $\mathbb{F}_{p}$-algebra, then this group is annihilated by $p^{r}$. For a general ring $k$, we must instead consider the group $\operatorname{TR}_{q-\lambda}^{r}\left(k ; p, \mathbb{Z} / p^{v}\right)$ which is annihilated by $p^{v}$. It follows that the iterated Frobenius $F^{m}$ induces the zero map from the first term in the bottom line of (2.1) for $m+n$ to the first term in the bottom line of (2.1) for $n$. Hence, the canonical projection onto the second term of the bottom line of (2.1),

$$
\operatorname{TR}_{q}^{n}(A, B, I ; p) \rightarrow \bigoplus_{r=1}^{n-1} \bigoplus_{d \in I_{p}} \operatorname{TR}_{q-\lambda_{p^{r-1} d}}^{r}(k ; p)
$$

is an isomorphism of pro-abelian groups. Here, we recall, the structure map in the limit system on the left-hand side is the Frobenius map and in the limit system on the right-hand side is the canonical projection. The group $\mathrm{TR}_{q-\lambda_{p^{r-1} d}}^{r}(k ; p)$ is zero, if $q<\operatorname{dim}_{\mathbb{R}}\left(\lambda_{d}\right)=2 d$, and hence, the limit group is the product

$$
\mathrm{TF}_{q}(A, B, I ; p) \stackrel{\sim}{\longrightarrow} \prod_{r \in \mathbb{N}} \prod_{d \in I_{p}} \operatorname{TR}_{q-\lambda_{p^{r-1} d}}^{r}(k ; p) .
$$

We can now evaluate the bi-relative topological cyclic homology groups that are given by the following long-exact sequence.

$$
\cdots \rightarrow \mathrm{TC}_{q}(A, B, I ; p) \rightarrow \mathrm{TF}_{q}(A, B, I ; p) \stackrel{R-\mathrm{id}}{\longrightarrow} \mathrm{TF}_{q}(A, B, I ; p) \rightarrow \ldots
$$

Indeed, under the isomorphism (2.2), the map $R$ corresponds to the endomorphism of the product on the right-hand side of (2.2) that is induced from the map

$$
R: \operatorname{TR}_{q-\lambda_{p^{r-1} d}}^{r}(k ; p) \rightarrow \mathrm{TR}_{q-\lambda_{p^{r-2} d}}^{r-1}(k ; p) .
$$


Hence, the kernel of the map $R$ - id in (2.2) is identified with the limit

$$
\prod_{d \in I_{p}} \lim _{R} \mathrm{TR}_{q-\lambda_{p^{r-1} d}}^{r}(k ; p)
$$

and the cokernel is identified with the corresponding derived limit. The following Lemma 2.3 shows, in particular, that the limit system satisfies the Mittag-Leffler condition. Hence, the derived limit vanishes and we obtain an isomorphism

$$
\mathrm{TC}_{q}(A, B, I ; p) \stackrel{\sim}{\longrightarrow} \prod_{d \in I_{p}} \lim _{R} \operatorname{TR}_{q-\lambda_{p^{r-1} d}}^{r}(k ; p)
$$

as stated in Thm. B.

Lemma 2.3. The restriction map

$$
R: \mathrm{TR}_{q-\lambda_{p^{r-1} d}}^{r}(k ; p) \rightarrow \mathrm{TR}_{q-\lambda_{p^{r-2} d}}^{r-1}(k ; p)
$$

is an isomorphism, for $q<2 p^{r-1} d$.

Proof. We recall from [13, Thm. 2.2] that there is a long-exact sequence $\cdots \rightarrow \mathbb{H}_{q}\left(C_{p^{r-1}}, T(k) \wedge S^{\lambda_{p^{r-1} d}}\right) \rightarrow \mathrm{TR}_{q-\lambda_{p^{r-1} d}}^{r}(k ; p) \stackrel{R}{\rightarrow} \operatorname{TR}_{q-\lambda_{p^{r-2} d}}^{r-1}(k ; p) \rightarrow \cdots$

and that the left-hand groups are given by a spectral sequence

$$
E_{s, t}^{2}=H_{s}\left(C_{p^{r-1}}, \mathrm{TR}_{t-\lambda_{p^{r-1} d}}^{1}(k ; p)\right) \Rightarrow \mathbb{H}_{s+t}\left(C_{p^{r-1}}, T(k) \wedge S^{\lambda_{p^{r-1} d}}\right) .
$$

The groups in the $E^{2}$-term do not depend on the representation $\lambda_{p^{r-1} d}$ beyond its dimension, and they are zero if $t<\operatorname{dim}_{\mathbb{R}}\left(\lambda_{p^{r-1} d}\right)=2 p^{r-1} d$. It follows that the map $R$ is an isomorphism, if $q<2^{p^{r-1}} d$ as stated.

\section{§3. Regular $\mathbb{F}_{p}$-algebras}

Let $k$ be a regular $\mathbb{F}_{p}$-algebra. The structure of the groups $\operatorname{TR}_{q-\lambda}^{n}(k ; p)$ that occur on the right-hand side of the statement of Thm. B of the introduction is given by [14, Thm. 2.2.2], but see also [11, Thm. 11]. If $\lambda$ is a finite dimensional complex $\mathbb{T}$-representation, we define

$$
\ell_{r}=\ell_{r}(\lambda)=\operatorname{dim}_{\mathbb{C}}\left(\lambda^{C_{p^{r}}}\right)
$$

and $\ell_{-1}=\infty$ such that we have a descending sequence

$$
\infty=\ell_{-1} \geqslant \ell_{0} \geqslant \ell_{1} \geqslant \cdots \geqslant \ell_{r} \geqslant \ell_{r+1} \geqslant \cdots \geqslant \ell_{\infty}=\operatorname{dim}_{\mathbb{C}}\left(\lambda^{\mathbb{T}}\right) .
$$

Then the following result is [14, Thm. 2.2.2]. 
Theorem 3.1. Let $k$ be a regular $\mathbb{F}_{p}$-algebra, and let $\lambda$ be a finite dimensional complex $\mathbb{T}$-representation. There is a canonical isomorphism of abelian groups

$$
\bigoplus W_{s} \Omega_{k}^{q-2 m} \stackrel{\sim}{\longrightarrow} \mathrm{TR}_{q-\lambda}^{n}(k ; p)
$$

where the sum runs over all integers $m \geqslant \ell_{\infty}$, and where $s=s(n, m, \lambda)$ is the unique integer such that $\ell_{n-s} \leq m<\ell_{n-1-s}$. The group $W_{s} \Omega_{k}^{j}$ is understood to be zero for non-positive integers $s$.

Remark 3.2. It appears to be an important problem to determine the structure of the $R O(\mathbb{T})$-graded equivariant homotopy groups

$$
\operatorname{TR}_{\alpha}^{n}(k ; p)=\left[S^{\alpha} \wedge\left(\mathbb{T} / C_{p^{n-1}}\right)_{+}, T(k)\right]_{\mathbb{T}}
$$

for a general virtual $\mathbb{T}$-representation $\alpha$. The precise definition of $R O(G)$ graded equivariant homotopy groups in given in [17, Appendix]. One might well hope that the $R O(\mathbb{T})$-graded equivariant homotopy groups $\operatorname{TR}_{\alpha}^{n}(k ; p)$ admit an algebraic description similar to that of the $\mathbb{Z}$-graded equivariant homotopy groups $\operatorname{TR}_{q}^{n}(k ; p)$ given in $[10, \mathrm{Thm} . \mathrm{B}]$.

We can now complete the proof of Thm. A of the introduction.

Proof of Theorem A. It suffices by Thm. B to show that for positive integers $d$ prime to $p$, there is a canonical isomorphism of abelian groups

$$
\bigoplus_{m \geqslant 0} W_{s} \Omega_{k}^{q-2 m} \stackrel{\sim}{\longrightarrow} \lim _{R} \operatorname{TR}_{q-\lambda_{p^{r-1} d}}^{r}(k ; p)
$$

where $s=s(m, d)$ is the unique integer that satisfies $p^{s-1} d \leq m<p^{s} d$. It follows from Lemma 2.3 that the canonical projection

$$
\lim _{R} \operatorname{TR}_{q-\lambda_{p^{r-1} d}}^{r}(k ; p) \rightarrow \operatorname{TR}_{q-\lambda_{p^{n-1} d}}^{n}(k ; p)
$$

is an isomorphism for $q<\operatorname{dim}_{\mathbb{R}}\left(\lambda_{p^{n}} d\right)=2 p^{n} d$. But for $\lambda=\lambda_{p^{n} d}$ we have

$$
\ell_{-1}=\infty \geqslant \ell_{0}=p^{n} d \geqslant \ell_{1}=p^{n-1} d \geq \cdots \geqslant \ell_{n}=d \geqslant \ell_{n+1}=\ell_{\infty}=0 .
$$

Hence, Thm. 3.1 gives a canonical isomorphism of abelian groups

$$
\bigoplus_{m \geqslant 0} W_{s} \Omega_{k}^{q-2 m} \stackrel{\sim}{\longrightarrow} \mathrm{TR}_{q-\lambda_{p^{n-1} d}}^{n}(k ; p)
$$

where $s=s(n, m, \lambda)$ is the minimum of $n$ and the unique positive integer $t$ that satisfies $p^{t-1} d \leqslant m<p^{t} d$. The statement follows. 
Remark 3.3. We conclude this paper with a conjecture on the relationship of the $K$-groups of the rings $k[x, y] /(x y)$ and $k[t] /\left(t^{2}\right)$. The element $f=x-y$ of the ring $A=k[x, y] /(x y)$ is a non-zero-divisor with quotient ring $A / f A=k[t] /\left(t^{2}\right)$. It follows that as an $A$-module $A / f A$ has projective dimension 1 , and hence we have a push-forward map on the associated $K$-groups

$$
i_{*}: K_{q}\left(k[t] /\left(t^{2}\right)\right) \rightarrow K_{q}(k[x, y] /(x y)) .
$$

The additivity theorem implies that the image of the map $i_{*}$ is contained in the subgroup $K_{q}(A, I)$. In particular, we obtain an induced push-forward map

$$
i_{*}: K_{q}\left(k[t] /\left(t^{2}\right),(t)\right) \rightarrow K_{q}(k[x, y] /(x y),(x, y)) .
$$

For $k$ a regular $\mathbb{F}_{p^{-}}$algebra, the relative $K$-groups on the right and left-hand sides were evaluated in Thm. A and [14, Thm. A], respectively. On the one hand, there is a natural long-exact sequence of abelian groups

$$
\cdots \rightarrow K_{q}\left(k[t] /\left(t^{2}\right),(t)\right) \stackrel{\partial}{\rightarrow} \bigoplus_{m \geqslant 1} \mathbf{W}_{m} \Omega_{k}^{q-2 m} \stackrel{V_{2}}{\longrightarrow} \bigoplus_{m \geqslant 1} \mathbf{W}_{2 m} \Omega_{k}^{q-2 m} \rightarrow \cdots
$$

and on the other hand, there is a canonical isomorphism of abelian groups

$$
I: \bigoplus_{m \geqslant 1} \mathbf{W}_{m} \Omega_{k}^{q-2 m} \stackrel{\sim}{\longrightarrow} K_{q}(k[x, y] /(x y),(x, y)) .
$$

We conjecture that the composite $I \circ \partial$ is equal to the push-forward map $i_{*}$.

\section{REFERENCES}

[1] A. A. Beilinson, Higher regulators of modular curves, Applications of algebraic $K$ theory to Algebraic Geometry and Number Theory (Boulder, CO, 1983), Contemp. Math., vol. 55, Amer. Math. Soc., Providence, RI, 1986, pp. 1-34.

[2] S. Bloch and H. Esnault, An additive version of higher Chow groups, Ann. Sci. École Norm. Sup. 36 (2003), 463-477.

[3] M. Bökstedt, W.-C. Hsiang, and I. Madsen, The cyclotomic trace and algebraic Ktheory of spaces, Invent. Math. 111 (1993), 465-540.

[4] G. Cortiñas, The obstruction to excision in $K$-theory and in cyclic homology, Invent. Math. 164 (2006), 143-173.

[5] R. K. Dennis and M. I. Krusemeyer, $K_{2}(A[X, Y] /(X Y))$, a problem of Swan, and related computations, J. Pure Appl. Alg. 15 (1979), 125-148.

[6] T. Geisser and L. Hesselholt, Bi-relative algebraic K-theory and topological cyclic homology, Invent. Math. 166 (2006), 359-395. 
[7] S. Geller, L. Reid, and C. A. Weibel, The cyclic homology and K-theory of curves, Bull. Amer. Math. Soc. 15 (1986), no. 2, 205-208.

[8] The cyclic homology and K-theory of curves, J. reine angew. Math. 393 (1989), 39-90.

[9] J. A. Guccione, J. J. Guccione, M. J. Redondo, and O. E. Villamayor, Hochschild and cyclic homology of hypersurfaces, Adv. Math. 95 (1992), 18-60.

[10] L. Hesselholt, On the p-typical curves in Quillen's K-theory, Acta Math. 177 (1997), $1-53$.

[11] K-theory of truncated polynomial algebras, Handbook of $K$-theory, Springer-Verlag, New York, 2005.

[12] L. Hesselholt and I. Madsen, Cyclic polytopes and the K-theory of truncated polynomial algebras, Invent. Math. 130 (1997), 73-97.

[13] On the K-theory of finite algebras over Witt vectors of perfect fields, Topology 36 (1997), 29-102.

[14] On the K-theory of nilpotent endomorphisms, Homotopy methods in algebraic topology (Boulder, CO, 1999), Contemp. Math., vol. 271, Amer. Math. Soc., Providence, RI, 2001, pp. 127-140.

[15] On the de Rham-Witt complex in mixed characteristic, Ann. Sci. École Norm. Sup. 37 (2004), 1-43.

[16] L. Illusie, Complexe de de Rham-Witt et cohomologie cristalline, Ann. Sci. École Norm. Sup. 12 (1979), 501-661.

[17] L. G. Lewis and M. A. Mandell, Equivariant universal coefficient and Künneth spectral sequences, Proc. London Math. Soc. 92 (2006), 505-544.

[18] J.-L. Loday, Cyclic homology, Grundlehren der mathematischen Wissenschaften, vol. 301, Springer-Verlag, New York, 1992.

[19] K. Rülling, The generalized de Rham-Witt complex over a field is a complex of zerocycles, J. Algebraic Geom. 16 (2007), 109-169.

[20] C. A. Weibel, Mayer-Vietoris sequences and $\bmod p K$-theory, Algebraic $K$-theory, Part I (Oberwolfach, 1980), Lecture Notes in Math., vol. 966, Springer-Verlag, New York, 1982, pp. 390-407.

Massachusetts Institute of Technology

Cambridge,Massachusetts

larsh@math.mit.edu

Nagoya University, Nagoya, Japan

larsh@math.nagoya-u.ac.jp 